\title{
A STUDY ON THE WACF SLOT SUB-ARRAY ANTENNA
}

\author{
Sun-yeob Kim* \\ Communication-Information Department, Namseoul University, Korea \\ sunyeobkim@gmail.com
}

\begin{abstract}
G network system is expected to have an exponential increase in terms of the number of small cell base stations due to finer cell segmentation than existing ones. Connecting all such base stations to a wired interface through optical cables, a conventional method, would be costly and time-consuming in the installation process, which leads to interest in building a wireless backhaul network system. To transmit a large amount of data in high quality through a wireless backhaul network, a frequency channel with wide bandwidth is required, hence millimeter wave (mmWave) band (30GHz $\sim 300 \mathrm{GHz})$ could be effectively implemented. Particularly, mmWave has following advantages: it is possible to reduce the size and weight of the related equipment including an antenna; high directivity of the wave makes it strong against the surrounding interference; it features strong security and easy frequency reuse. Based on these, this paper focuses on a slot sub-array antenna with WACF (Waveguide aperture-coupled feed) structure that can be implemented into a wireless backhaul network in which high capacity and Gbps data transmission is possible. A WACF $32 \times 32$ slot sub-array antenna that features broadband and high gain was designed and fabricated to be implemented into the $\mathrm{V}$-band in the spectrum between $57 \mathrm{GHz}$ and $66 \mathrm{GHz}$ frequencies. The antenna is structured in a laminated form, composed of a radiating part (outer groove, slot, cavity), a coupled aperture, and feeds. Analytical test and simulation were performed using HFSS (High frequency structure simulator), which is based on the 3-D FEM (Finite element method). For the fabrication of the antenna, each layer of the antenna was first created using precisioncontrolled milling machine and then each layer was assembled after silver-plating with through conductive epoxy. As a result of analyzing the characteristics of the antenna in the mmWave dedicated anechoic chamber, the return-loss in the target frequency band was less than $-10 \mathrm{~dB}$, VSWR $<2.0$ and wide bandwidth ranged up to approximately $16 \%$.
\end{abstract}

Keywords - Antenna, Aperture, Bandwidth, Coupling, Waveguide

\section{INTRODUCTION}

With the emergence of big data as mobile smart devices and services evolve to new forms around the world, data transmission volumes are rapidly increasing every year. As the data transmission volumes are expected to increase more than 1,000 times in 2020 compared to current $4 \mathrm{G}$ networks, a variety of new wireless communication technologies that increase spectral efficiency with advanced modulation systems have been researched and currently being implemented to cope with data capacity increase, such as Massive MIMO (Multi-input, Multi-output) technology which implements multiple transmission

Received: May 8, 2019

Reviewed: July 19, 2019

Accepted: August 2, 2019

* Corresponding Author 
and reception antennas to base stations, NOMA (Non-orthogonal multiple access) and UWB (Ultra-wideband) technologies for bandwidth efficiency, and etc.

Meanwhile, the next generation $5 \mathrm{G}$ network system is expected to have an exponential increase in the number of small cell base stations due to finer cell segmentation than existing ones. Since connecting all of these small cell base stations through optical fiber cables is time-consuming in the installation process and causes a heavy financial strain, building a wireless backhaul network system, much more efficient in a flexible operation of cells, is more preferred today. Building a backhaul network system helps the operator cope with rapidly increasing data transmission demand flexibly as it can employ not only conventional stationary base stations, but also the mobile base stations.

To transmit a large amount of data in high quality through a wireless backhaul network, a frequency channel with wide bandwidth is required, hence millimeter wave (mmWave) band $(30 \mathrm{GHz} \sim 300 \mathrm{GHz})$ could be an effective mean. Particularly, mmWave has following advantages: it is possible to reduce the size and weight of the related equipment including antenna; high directivity of the wave makes it strong against the surrounding interference; it features higher security and easy frequency reuse. However, since the mmWave band has an inherent characteristic that the loss in free space is higher than that of other frequency bands, research on this matter needs to be preceded. Signals in mmWave are normally damped by atmospheric oxygen, vapor, and rainfall. While this damping causes less smooth long-range communication, it can be rather utilized usefully in a short-range small cell environment where LOS (Line-of-sight) is secured. That is, in the case of the $60 \mathrm{GHz} \mathrm{V}$ band $(50 \mathrm{GHz} \sim 75 \mathrm{GHz})$, as the wireless link distance gets longer, the radiation power of the carrier frequency is drastically lowered to the point where it does not interfere with other surrounding links of the same frequency. Therefore, frequency reuse is easy because there is no hindrance in forming various wireless links and communicating even if the same frequency channel is concurrently used in close proximity.

Accordingly, there have been various attempts to develop a wireless device to be implemented into the $60 \mathrm{GHz}$ because the frequency band is allocated as 'unlicensed frequency bands', so that the use of the frequency is free. In addition, it is possible to transmit data of several tens of Gbps with a simple demodulating device without using a complicated and expensive modem device to compress data since the frequency bandwidth (in the spectrum between $57 \mathrm{GHz}$ and $66 \mathrm{GHz}$ ) is $9 \mathrm{GHz}$.

The mmWave band is now becoming the central frequency resource for the nextgeneration wireless communications around the world. In Korea, commercialization of components and systems for mmWave above $50 \mathrm{GHz}$ has not yet been fully achieved, except for only a small number of specialized companies in the industry, compared to mobile communications technologies below $6 \mathrm{GHz}$ and microwave communication technologies below $30 \mathrm{GHz}$. The most point-to-point communication mmWave antennas currently implemented in the field, in particular, are imported.

Thus, this paper proposes a WACF (Waveguide Aperture-Coupled Feed) slot sub-array antenna that features broadband and high gain, which to be implemented into the wireless backhaul system in the $60 \mathrm{GHz}$ V-band where high-capacity Gbps data transmission is provided.

\section{WACF SLOT SUB-ARRAY ANTENNA}

The single model analysis of the $60 \mathrm{GHz} \mathrm{V}$-band WACF slot sub-array antenna proposed in this paper is based on numerical analysis methods such as the 3-D FEM described in the previous section. HFSS, a commercial simulator using algorithms with improved calculation methods of the sides of tetrahedron for accurate and fast analysis on various models, was used for analysis.

Some of the major characteristics of the proposed WACF slot sub-array antenna are: it minimizes mutual coupling between adjacent radiating slots that can occur in antenna 
arraying by applying outer groove on the radiating slot; increases antenna gains like horn antennas; broadens the bandwidth reflection by adjusting the outer groove width. Therefore, the analysis derives results by comparing the characteristics of antenna returnloss and gain with the features of the mutual coupling between adjacent devices during subarray under two different conditions: when the outer groove was applied and not.

The proposed antenna consists of three layers as shown in Figure 1: the first layer is composed of a cavity for the outer groove, radiating slots, and sub-array; the second layer is a signal coupler that consists of feed-waveguide and coupled aperture; the third layer consists of a waveguide feeds that distributes signals to each coupled aperture.

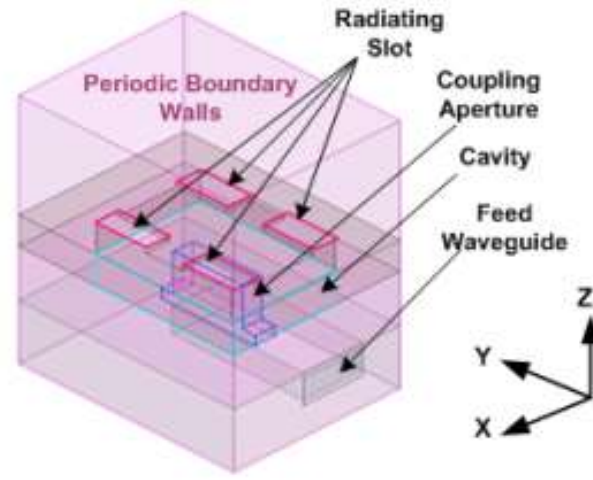

(a) $2 \times 2$ Sub-arry unit mode (Without Groove )

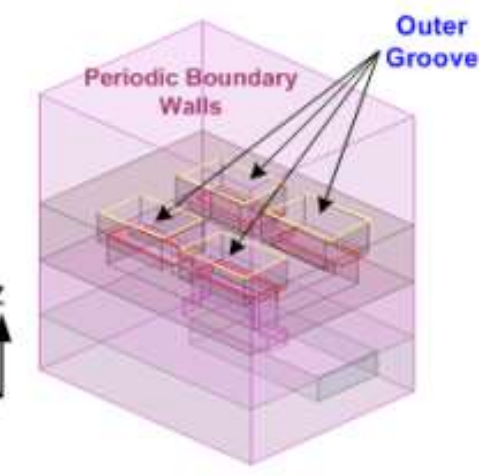

(b) $2 \times 2$ Sub-arry unit model (With Groove)

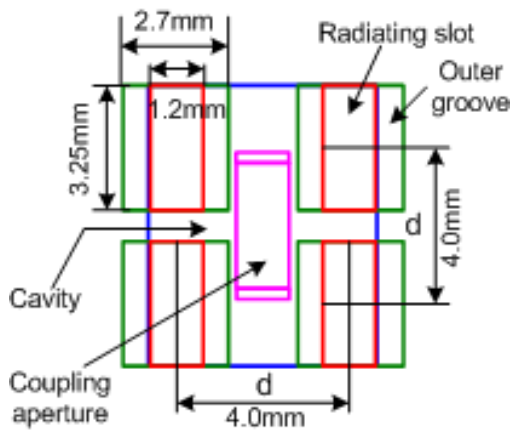

(c ) Radiating slot size \& spacing

Fig. 1 WACF $2 \times 2$ slot sub-array antenna unit model

In applying the basic design parameters for analysis, the center frequency of the designed antenna was set to $62 \mathrm{GHz}$, and since the radiating slot has a complementary characteristic with the half-wave dipole antenna, the slot length was set to $3.25 \mathrm{~mm}, \lambda \mathrm{g} / 2$, so as to resonate in the desired frequency band. In addition, the spacing between the radiation elements should be optimized to $0.84 \lambda 0$ due to the characteristics of the sub-array structure, which may cause a mutual coupling when the distance between slots $d$ is $d \leq \lambda 0$, or grating-lobe may occur when $d \geq \lambda 0$. The cavity composing the radiating part is structured in a way that four slots are in correspondence with one coupled aperture. Since it collects signals at the time of transmission and reception, it was set to $\lambda 0 / 4$ to minimize signal loss from impedance matching and reflected waves. Based on what was described above, the length of the radiating slot was set to $3.25 \mathrm{~mm}$, width to $1.2 \mathrm{~mm}$; the length of the outer groove set equal to that of the radiating slot while the width was set to $2.7 \mathrm{~mm}$ to extend the reflection bandwidth.

Among the parameters that determine the characteristics of the slot antenna, the size of the slot is related to the resonant frequency and bandwidth of the antenna. That is, the resonant frequency of the antenna is affected by the slot length while the width has an effect 
on the reflection bandwidth. Adjusting slot width should be conducted carefully to avoid a mutual coupling between adjacent slots. Once mutual coupling occurs, the current distribution between adjacent slots begin to affect each other, resulting in the input impedance and radiation pattern of the antenna also affected eventually.

The proposed antenna in this study broadens the bandwidth by implementing an outer groove on the radiating slot, allowing each radiating slot to maintain radiating characteristics, thereby achieving a high gain. The outer groove has a function of effectively preventing a mutual coupling that occurs during antenna array, hence improving the overall characteristics of the antenna.

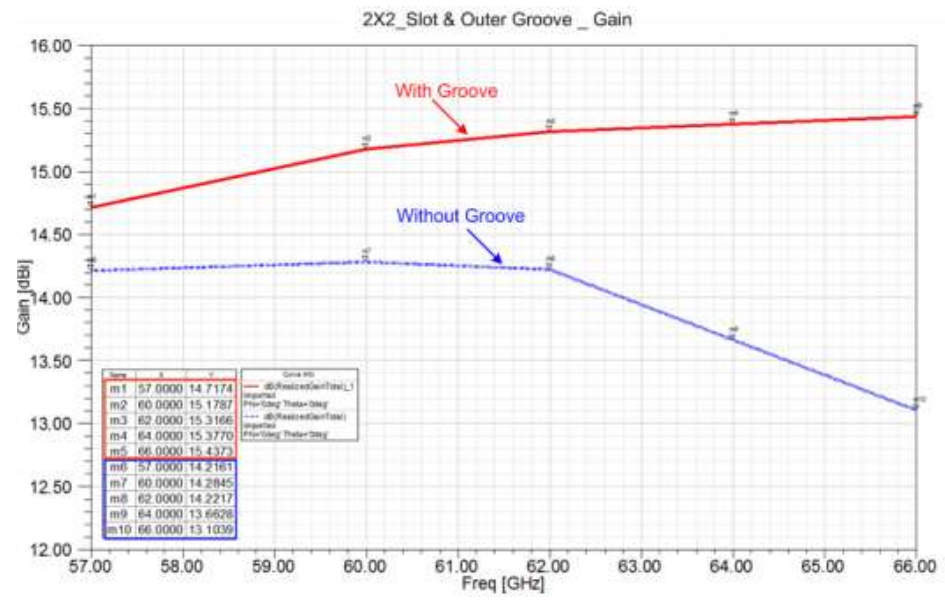

Fig. 2 Gain of WACF $2 \times 2$ slot sub-array antenna

Figure 2 shows the characteristics of the gain from two antennas. The antenna with outer groove has a high gain; it has a gain of $15.3 \mathrm{dBi}, 1.1 \mathrm{dBi}$ higher than the one without outer groove in the center frequency of $62 \mathrm{GHz}$ and $2.3 \mathrm{dBi}$ higher in the frequency of $66 \mathrm{GHz}$. It confirms that the outer groove works as a horn antenna improving the gain as well as is related to the reflection bandwidth and mutual coupling as described above.

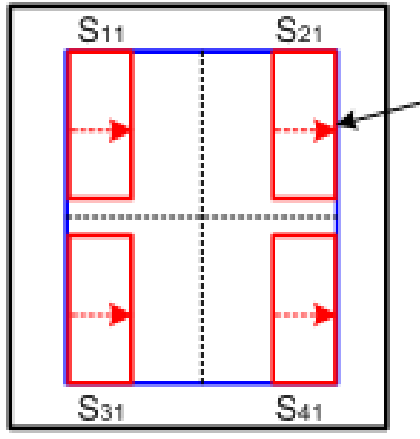

(a) Without groove structure

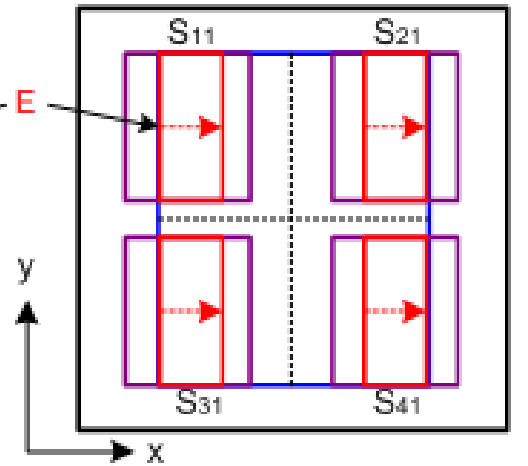

(b) With a groove structure

Fig. 3 Configurations to examine the mutual-coupling among the $2 \times 2$ radiation slot

Figure 3 is a $2 \times 2$ slot sub-array antenna configuration shown to compare mutual coupling between radiating slots. Both the model without the outer groove and the one with the outer groove were applied with regular boundaries and the distance between the radiating slots was arranged at intervals of $0.84 \lambda 0$ in order to simulate the models in a 2$\mathrm{D}$ infinite plane array condition. 


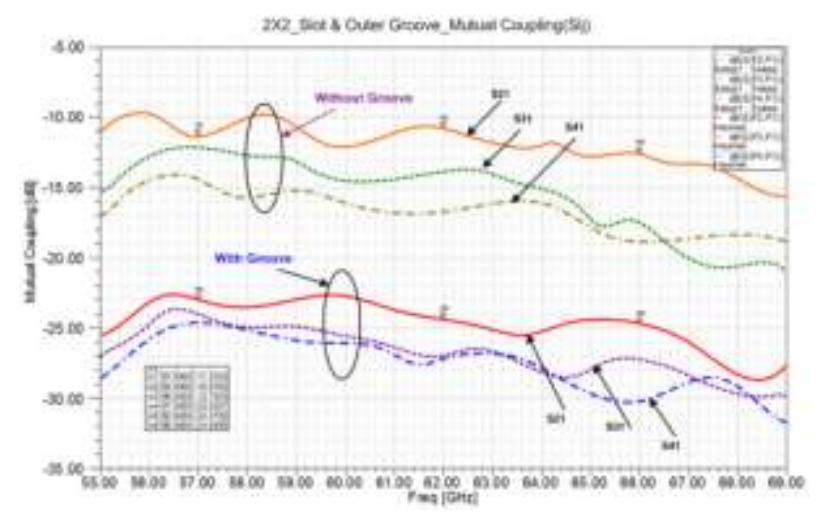

Fig. 4 With \& without the groove between each 4-slot mutual-coupling characteristics

Figure 4 shows the state of mutual coupling between each radiating slot with and without the outer groove. As shown in the figure, without the outer groove, the mutual coupling degree between S11 and S21, which is in the same direction with E-field on the X-axis, is $55.8 \mathrm{GHz}$, showing strong coupling characteristics with $-10 \mathrm{~dB}$ at $58.4 \mathrm{GHz}$. On the other hand, the one with the outer groove shows the degree of mutual coupling between S11 and $\mathrm{S} 21$ as $-22.5 \mathrm{~dB}$.

That is, it confirms that the mutual coupling is restrained as much as approximately 12 $\mathrm{dB}$ without the outer groove. In addition, it shows that the mutual coupling between S11 and S31 (y-axis) in the vertical direction and S41 in the diagonal direction is restrained to below $-25 \mathrm{~dB}$. What these results indicate is that the radiating slot's perimeter of the proposed antenna with the outer groove is affected less by the characteristics of mutual coupling than when there is no outer groove as if the characteristics of every single radiating element are not changed regardless of where those elements are positioned at the array antenna.

\section{WACF SLOT SUB-ARRAY ANTENNA CONFIGURATION}

Figure 5 is a single model for a $2 \times 2$ slot sub-array antenna configuration applied with regular boundary walls to show the effects of mutual coupling between radiating elements in a 2-D infinite plane array. The center frequency of the designed antenna was set to $62 \mathrm{GHz}$ to have the frequency band in the spectrum of $57 \mathrm{GHz}$ and $66 \mathrm{GHz}$. The slot spacing including the outer groove was set at 4.0, $0.84 \lambda 0$, and the design and simulation were conducted using HFSS v.15 based on the 3-D finite element method. As mentioned in Chapter 3, the radiating slot has a complementary characteristic with the half-wave dipole antenna, therefore the length was set to $3.25 \mathrm{~mm}, 0.5 \lambda \mathrm{g}$. First, it studies the characteristics of the reflection bandwidth according to the variation of slot width for designing the optimized radiating part (outer groove, radiating slot) and then checks the characteristics of frequency band and return-loss according to the variation of groove width after implementing the outer groove. Figure 6 shows the characteristics of return-loss and frequency band according to the variation of radiating slot width (WS) in WACF 2 × 2 slot sub-array antenna configuration without the outer groove. When the slot width is small, a strong resonance occurs toward the low-frequency side showing the gradual increase in the frequency separation characteristic. When the width increases, the overall return-loss increases, and the frequency separation becomes gentle. 


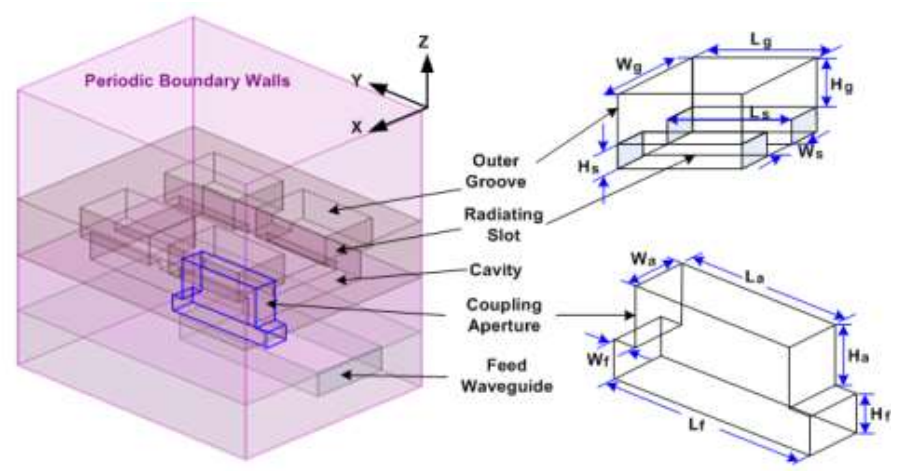

Fig. 5 Design of WACF $2 \times 2$ slot sub-array antenna

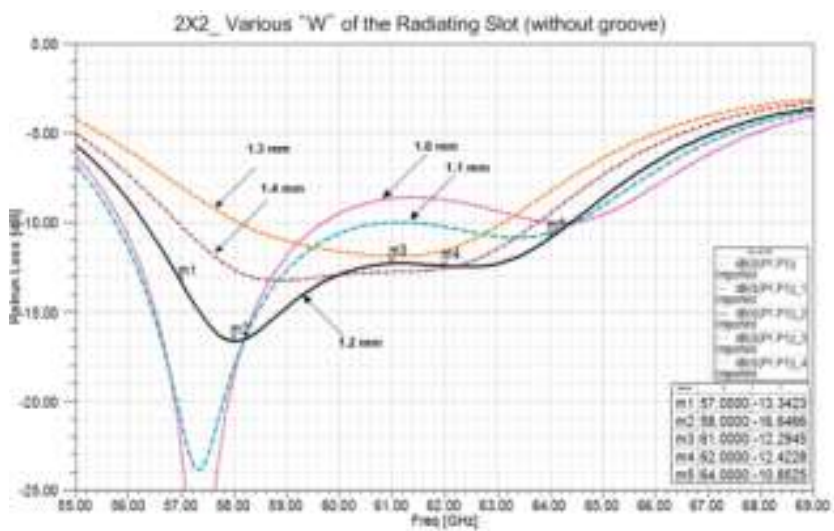

Fig. 6 Return loss due to slot width $\left(W_{S}\right)$ variation (without groove)

Since the target frequency band of the antenna in this study ranges between $57 \mathrm{GHz}$ and $66 \mathrm{GHz}$ and the VSWR $<2.0$, the measurement results are not satisfactory but the slot width of $1.2 \mathrm{~mm}$ was applied to the design as the closest characteristic is shown at $1.2 \mathrm{~mm}$. Figure 7 shows the characteristics of return-loss and frequency according to the variation of the groove width after implementing the outer groove on the designed slots.

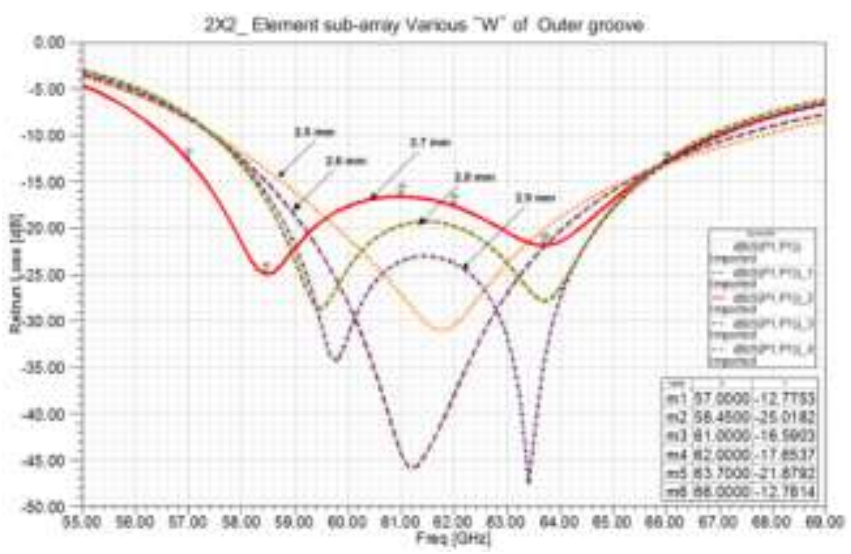

Fig. 6 Return loss due to outer groove width $\left(W_{g}\right.$ variation $)$

As shown in Figure 7, when the groove width is increased with the outer groove in the antenna, the return-loss characteristic is lowered and shows the stable characteristic by obtaining wide bandwidth. However, when the width is widened to $2.9 \mathrm{~mm}$, the return-loss decreases while the resonance frequency is shifted to a higher frequency band and the bandwidth is reduced. Therefore, the outer groove width was designed to be $2.7 \mathrm{~mm}$, which 
satisfies both the bandwidth and return-loss characteristics. The antenna design parameters in Table I show: the outer groove's length and width; radiating slot's length and width; and the size for the coupling aperture with the aperture-step form for the impedance matching with the feed-waveguide.

\begin{tabular}{|c||c|c|}
\hline \multicolumn{1}{|c||}{ Design } & \multicolumn{2}{|c|}{ Size $(\mathrm{mm})$} \\
\hline \multirow{2}{*}{ Radiation elements } & $\operatorname{Slot}\left(W_{S} \times L_{S} \times H_{S}\right)$ & $1.2 \times 3.25 \times 0.5$ \\
\cline { 2 - 3 } & Outer groove $\left(W_{g} \times L_{g} \times H_{g}\right)$ & $2.4 \times 3.25 \times 1.5$ \\
\hline \multirow{2}{*}{ Middle cavity } & $\operatorname{Cavity}\left(W_{c} \times L_{c} \times H_{c}\right)$ & $5.2 \times 7.2 \times 1.0$ \\
\hline \multirow{2}{*}{ Coupling aperture } & $\operatorname{Top}\left(W_{a} \times L_{a} \times H_{a}\right)$ & $1.1 \times 3.6 \times 1.0$ \\
\cline { 2 - 3 } & $\operatorname{Step}\left(W_{a} \times L_{a} \times H_{a}\right)$ & $1.1 \times 3.6 \times 2.0$ \\
\hline Width of wall & \multicolumn{2}{|c}{0.8} \\
\hline Slot spacing & \multicolumn{2}{|c}{4.0} \\
\hline
\end{tabular}

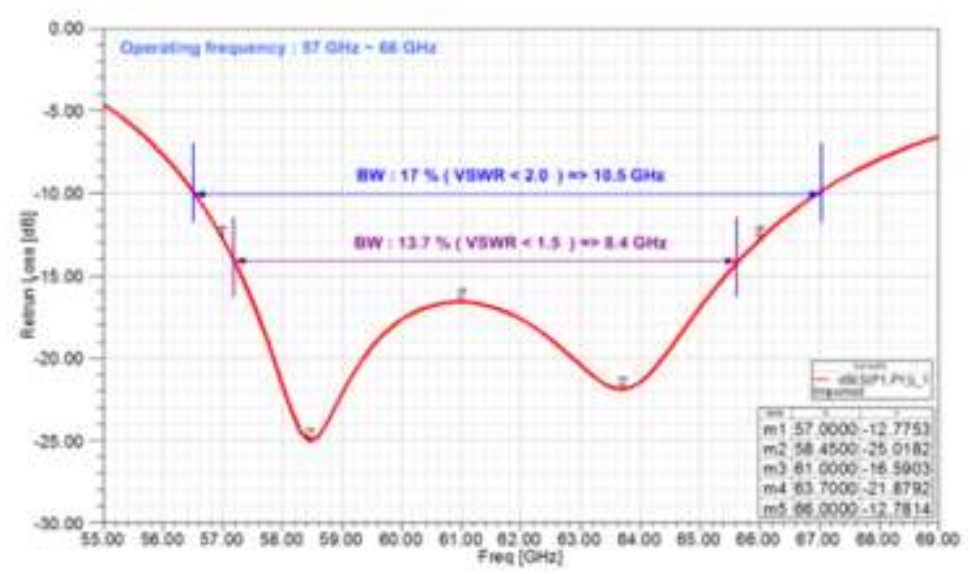

Figure 8 . Return loss $\&$ radiation pattern of the WACF $2 \times 2$ slot sub-array antenna

Figure 8 shows a WACF $2 \times 2$ slot sub-array antenna's return-loss, frequency band characteristics, and radiation pattern through simulation. It shows a bandwidth of $17 \%$ based on VSWR $<2.0$ and a gain of $15.3 \mathrm{dBi}$ at $62 \mathrm{GHz}$.

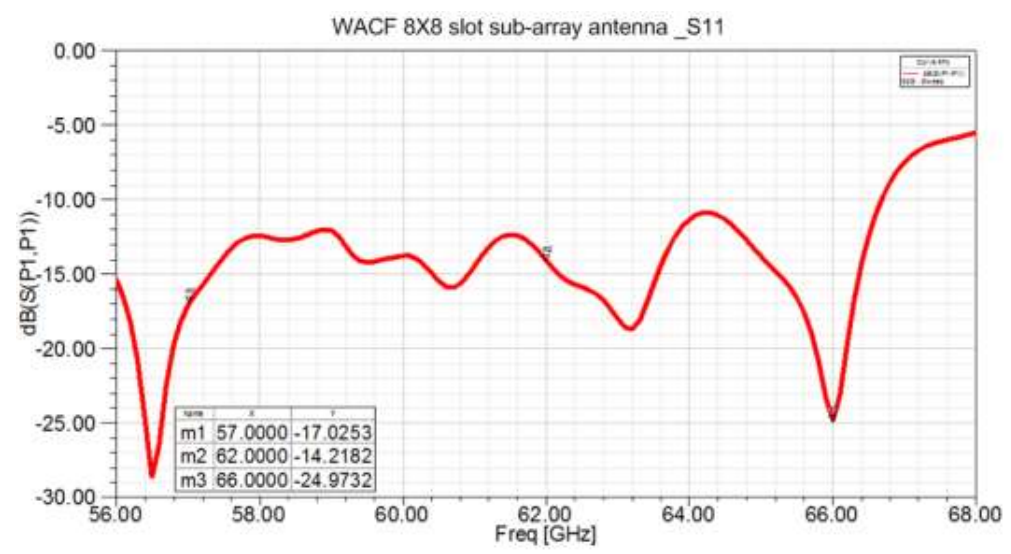

Figure 9. Return loss of WACF $8 \times 8$ slot sub-array antenna 
As shown in Figure 9, the return-loss characteristic of the WACF slot sub-array antenna shows broadband characteristics in general within the operating frequency range. It has the maximum return-loss of $-11 \mathrm{~dB}$ at $64.2 \mathrm{GHz}$ and the minimum $-28.7 \mathrm{~dB}$ at $56.5 \mathrm{GHz}$.

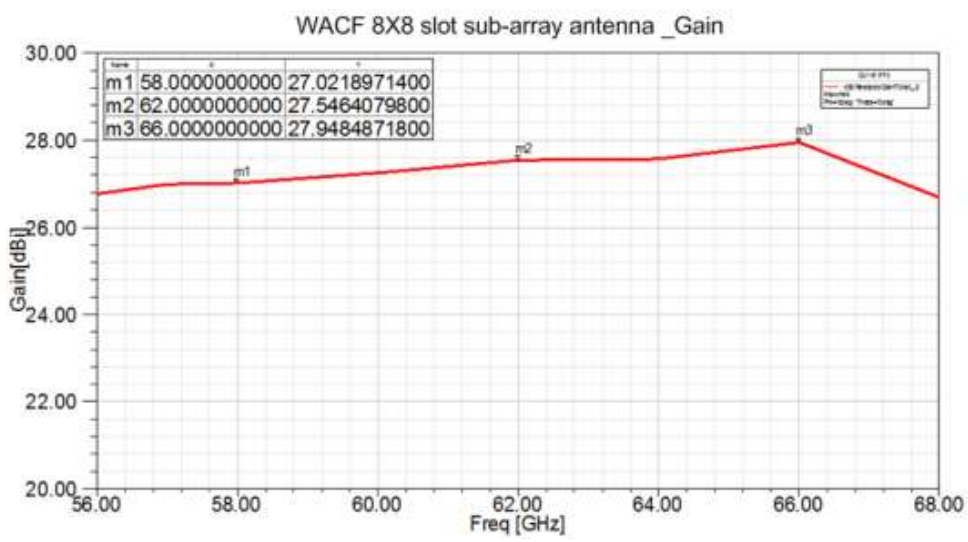

Figure 10. Gain of WACF $8 \times 8$ slot sub-array antenna

As shown in Figure 10, the gain is as high as $27.5 \mathrm{dBi}$ at $62 \mathrm{GHz}$ and $27.9 \mathrm{dBi}$ at $66 \mathrm{GHz}$, and the flatness of gain is stable within $1 \mathrm{~dB}$ as well.
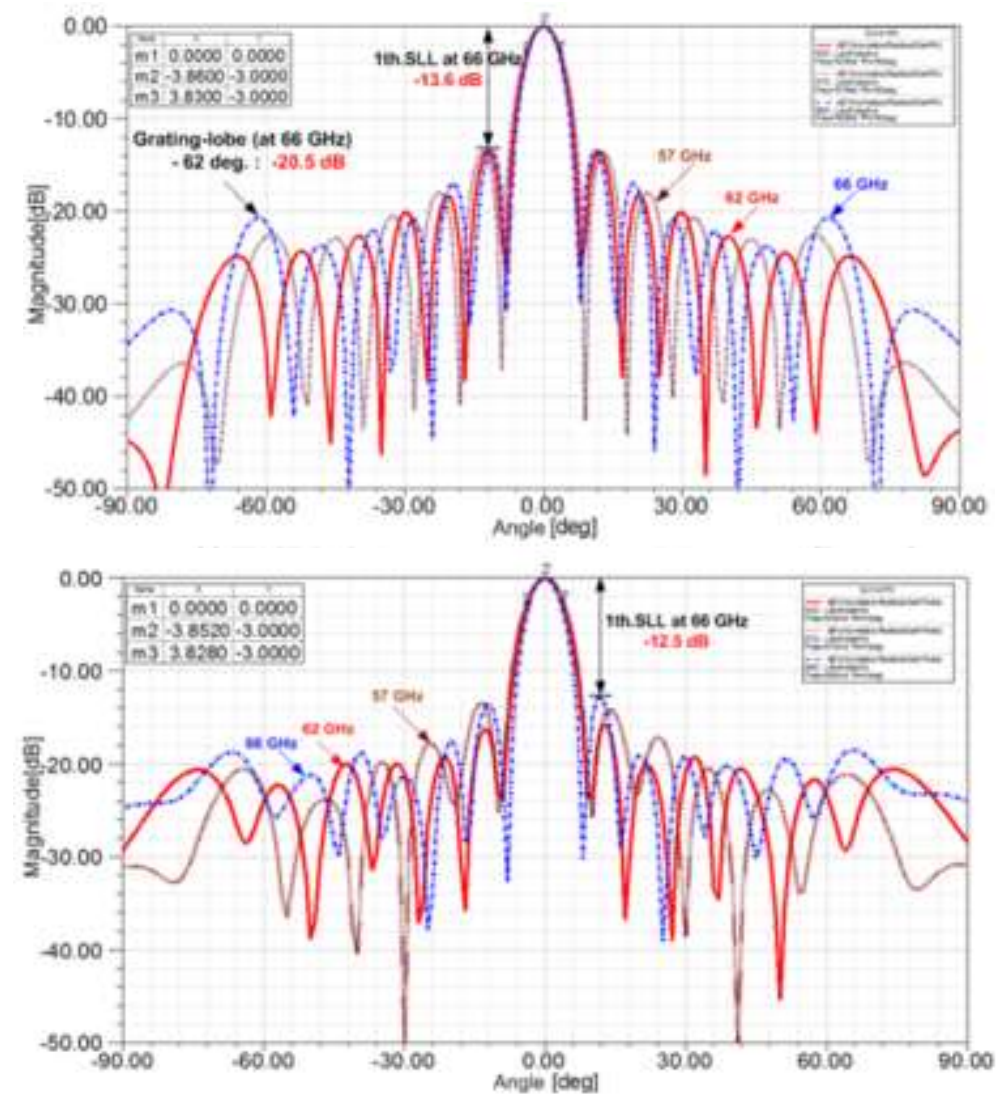

Figure 11. Radiation pattern of WACF $8 \times 8$ slot sub-array antenna

Figure 11 shows the radiation pattern characteristics of the WACF slot sub-array antenna: the $3 \mathrm{~dB}$ beam width at the E-plane is $7.69^{\circ}$; the primary side-lobe level is $-13.6 \mathrm{~dB}$ $(66 \mathrm{GHz})$; the grating-lobe level at $-62^{\circ}$ is restrained to $-20.5 \mathrm{~dB}$. In the H-plane: the $3 \mathrm{~dB}$ 
beam width is $7.68^{\circ}$; the maximum primary side-lobe level is $-12.5 \mathrm{~dB}$ showing a satisfactory characteristic.

\section{CONCLUSION}

As data transmission volumes are explosively growing due to the increase of various smart device use, this paper proposes a new high-gain broadband wireless communication system that can be implemented into backhaul equipment of the next-generation $5 \mathrm{G}$ network small cell stationary wireless communication system, which can transmit highcapacity data at Gbps. Each element composing the antenna was analyzed and their characteristics were also investigated through designing and fabricating processes.

The proposed WACF slot sub-array antenna was designed to operate in the $60 \mathrm{GHz} \mathrm{V}$ band, a mmWave; each radiating slot was implemented with the outer groove in an antenna array in order to improve gain while restraining the effects of mutual coupling between radiating slots. In addition, the feeds, which is one of the most essential elements of the antenna, adopt the waveguide corporate-feed network, which has little feed loss, for low return-loss and broadband characteristics. The $\mathrm{H}$-plane T-junction divider and $\mathrm{H}$-junction divider, which compose the feeds, were optimized to achieve low return-loss and equal power distribution to each output port in the $60 \mathrm{GHz}$ band using a septum and an iris.

\section{ACKNOWLEDGMENTS}

Funding for this paper was provided by Namseoul University.

\section{REFERENCES}

[1] Faragher, John Mack, "Bungalow and Ranch House: The Architectural Backwash of California", Western Historical Quarterly 32.2 (2001): 149-173.

[2] T. Zwick, D. Liu and B. P. Gaucher, "Broadband planar super substrate antenna for integrated millimeter wave transceivers", IEEE Trans. Antennas Propagation 54.10 (2006): 2790-2796.

[3] Min Wook Kang and Yun Won Chung, "A Novel Wireless TCP for 5G Mobile Network World Journal of Wireless Devices and Engineering”, 1.1(2017):1-6. http://dx.doi.org/10.21742/WJWDE.2017.1.1.01.

[4] T. Seki, N. Honma, K. Nishikawa, and K. Tsunekawa, "A 60-GHz multi-layer parasitic micro strip array antenna on LTCC substrate for system on package", IEEE Microwave Wireless Component Lett., 15.5 (2005): 339-341.

[5] Seyed Ali Razavi, Per-Simon Kildal, " $2 \times 2$ Slot Element for 60GHz Planar Array Antenna Realized on Two Doubled-sided PCBs Using SIW Cavity and EBG-type Soft Surface fed by Microstrip-Ridge Gap Waveguide", IEEE Trans., Antennas Propagation, 62.9(2014): 4564-4573.

[6] J. P. Webb, "Edge Elements and What They can do for you", IEEE Trans Magnetics., 29.2 (1993): 14601465 .

[7] A. Bagwari, J. Kanti and G. Singh Tomar, "Multiple Antennas Based Sensing Technique", International Journal of Urban Design for Ubiquitous Computing, 4.2(2016):41-50. http://dx.doi.org/10.21742/IJUDUC.2016.4.2.06.

[8] Chan-Soo Park, "Characteristics Analysis of the CM and DM Noise Separator in EMI", Journal of the Korea Academia-Industrial Cooperation Society, 17.5 (2016):49-55.

[9] M. Naveena Rama Krishna, M. Sivaji Ganesh, M. Prasanth Kumar Reddy, N. Venkatesh and K. Jagadeesh Babu, "A Dual-band Cross Shaped MIMO Antenna with Reduced Mutual Coupling”, International Journal of Communication Technology for Social Networking Services, 4.1(2016):1-8. http://dx.doi.org/10.21742/IJCTSNS.2016.4.1.01.

[10] R. Miniowitz, and J. P. Web, "Convariant-projection quadrilateral elements for the analysis of waveguide with sharp edges", IEEE Trans on microwave theory tech., MTT, 39.3 (1991): 501-505.

[11] K. Ise, K. Inoue and M. Koshiba, "Three-dimensional finite-element method with edge elements for electromagnetic waveguide dis-continuities", IEEE Trans., on Microwave Theory Tech. 39.8, (1991): 1289-1295.

[12] R. Gupta, A. Kalia, G. Gupta, A. Rajawat, S. Hak Gupta and M. R. Tripathy, "Performance Enhancement of an E-shaped Microstrip Patch Antenna loaded with Metamaterial", International Journal of Wireless and Mobile Communication for Industrial Systems, 3.2(2016):9-16 http://dx.doi.org/10.21742/IJWMCIS.2016.3.2.02.

[13] J-Ming Jin, John L, Volakis, "Electromagnetic Scattering by and Transmission Through a ThreeDimensional Slot in a Thick Conducting Plane”, IEEE Trans. on A\&P 39.4 (1991):543-550. 
[14] H. Lee, S. Ryu, S. Ryu, B. Kim, “A development of Folded Dipole Antenna for $26.5 \sim 29.5 \mathrm{MHz}$ Band for immunity test by portable transmitters on vehicle components", International Journal of Energy, Information and Communications, 9.5(2018):1-6 http://dx.doi.org/10.21742/IJEIC.2018.9.5.01.

[15] Tae-Hyun Lee, Chulho Yang, "A Study on Optimal Shape of Stent by Finite Element Analysis”, Journal of the Korea Academia-Industrial Cooperation Society 18.11(2017) 1-6.

[16] Miura, Y, Hirokawa, J, Zhang, M, Ando, M. "A 45ㄴinearly Polarized Hollow Waveguide Corporatefeed Slot Array Antenna in the 60GHz Band", IEEE Trans., Antennas Propagation, 60.8, (2012): 36403646.

[17] Per-Simon Kildal, " $2 \times 2$ Slot Element for $60 \mathrm{GHz}$ Planar Array Antenna Realized on Two Doubled-sided PCBs Using SIW Cavity and EBG-type Soft Surface fed by Microstrip-Ridge Gap Waveguide”, IEEE Trans., Antennas Propagation, 62.9(2014) 4564-4573. 